\title{
Paired whole exome and transcriptome analyses for the Immunogenomic changes during concurrent chemoradiotherapy in esophageal squamous cell carcinoma
}

Sehhoon Park ${ }^{1 \dagger}$, Je-Gun Joung ${ }^{2 \dagger}$, Yang Won Min ${ }^{3+}$, Jae-Yong Nam², Daeun Ryu ${ }^{2}$, Dongryul $\mathrm{Oh}^{4}$, Woong-Yang Park' ${ }^{2}$, Se-Hoon Lee ${ }^{1}$, Yoon La Choi ${ }^{5}$, Jin Seok Ahn' ${ }^{1}$, Myung-Ju Ahn', Keunchil Park and Jong-Mu Sun ${ }^{1 *}$

\begin{abstract}
Background: The immunogenomic changes triggered by concurrent chemoradiation therapy (CCRT), a standard neoadjuvant treatment for locally advanced esophageal squamous cell carcinoma (ESCC), are unknown. We aimed to analyze the early immunogenomic changes in ESCC induced by CCRT and to correlate them with clinical outcomes.

Methods: We collected biopsy samples from 40 patients with ESCC and the surgical candidates were treated with 5fluorouracil (5-FU)/Cisplatin and concurrent radiation therapy. Endoscopic biopsy was performed before and after one treatment cycle of 5-FU/Cisplatin and 5 to 18 fractions of radiation. We analyzed immunogenomic changes using paired whole-exome sequencing $(n=29)$ and paired whole-transcriptome sequencing (WTS, $n=23)$. Multiplex immunohistochemistry $(\mathrm{IHC})$ was conducted in four representative pair samples.
\end{abstract}

Results: Fourteen out of 23 WTS samples (60.8\%) showed increased immune scores after CCRT, as calculated by ESTIMATE. The rate of progression-free survival was higher in patients with increased immune scores compared with the remaining patients $(83.1 \%$ vs. $57.1 \%, p=0.25)$. Tumor mutation burden and neoantigen load were significantly reduced after CCRT $(p<0.001$ ). We observed no specific correlation with non-synonymous mutations and no changes in the single-nucleotide variant spectrum after CCRT. Post-CCRT samples were enriched in gene sets related to immune signaling pathways, such as interferon gamma signaling and CD28 co-stimulation. Multiplex IHC showed an incremental trend in the proportion of CD4 positive cells in cytokeratin positive region after CCRT. However, CD8, CD20, FOXP1, PD-L1 showed no definitive trend. Proportion of immune cells calculated by CIBERSORT, showed that significant increase in neutrophils after CCRT.

Conclusions: We have comprehensively analyzed the early immunogenomic changes induced in ESCC by CCRT and correlated them with clinical outcomes. Our results provide a potential basis for combining immunotherapy with CCRT for the treatment of ESCC.

Keywords: Esophageal neoplasms, Chemoradiotherapy, Immune checkpoint inhibitor

\footnotetext{
* Correspondence: jongmu.sun@skku.edu

† Sehhoon Park, Je-Gun Joung and Yang Won Min contributed equally as cofirst authors

${ }^{1}$ Division of Hematology-Oncology, Department of Medicine, Samsung

Medical Center, Sungkyunkwan University School of Medicine, 81 Irwon-ro,

Gangnam-Gu, Seoul 60351, Republic of Korea

Full list of author information is available at the end of the article
}

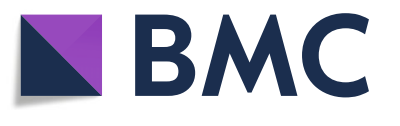

(c) The Author(s). 2019 Open Access This article is distributed under the terms of the Creative Commons Attribution 4.0 International License (http://creativecommons.org/licenses/by/4.0/), which permits unrestricted use, distribution, and reproduction in any medium, provided you give appropriate credit to the original author(s) and the source, provide a link to the Creative Commons license, and indicate if changes were made. The Creative Commons Public Domain Dedication waiver (http://creativecommons.org/publicdomain/zero/1.0/) applies to the data made available in this article, unless otherwise stated. 


\section{Background}

Esophageal cancer, the sixth leading cause of cancer-related deaths, is classified into two main histological subtypes: squamous cell carcinoma (SCC) and adenocarcinoma. Despite the decreased incidence of esophageal squamous cell carcinoma (ESCC) in Western countries, SCC remains prevalent in Asia, Africa, and South America [1]. The combined modality approach of preoperative concurrent chemoradiation therapy (CCRT) and surgery has shown superior clinical outcomes compared with surgery or chemotherapy alone for the treatment of locally advanced ESCC [2-4]. Despite the promising effects of preoperative CCRT, the challenge of cancer relapse after curative resection in patients with ESCC needs to be addressed.

Immune checkpoints are the downregulators of the anti-tumor immune response and their inhibitions are the good treatment strategy as cancer immunotherapy $[5,6]$. The synergistic effects of CCRT and immunotherapy have been tested in many clinical trials. In a recent trial, patients with locally advanced non-small cell lung cancer that were treated with durvalumab, an inhibitor of programmed cell death-ligand 1 (PD-L1), after CCRT showed prolonged progression-free survival (PFS) compared with patients that only underwent CCRT [7]. However, the underlying immunogenomic changes induced by CCRT in the tumor and its microenvironment remain unknown. Analysis of such changes may offer additional insights into the synergistic effects of immunotherapy and CCRT and also provide potential predictive or prognostic biomarkers [8].

Here, we conducted an immunogenomic analysis of CCRT-induced changes in ESCC cells using paired wholeexome sequencing (WES) and whole-transcriptome sequencing (WTS). We analyzed previously known immunogenomic markers, such as the proportion of immune cells in the tumor microenvironment $[9,10]$, somatic mutation profile $[11,12]$, expression of gene related to cytolytic activity $[13,14]$, the overall tumor mutation burden (TMB) [15-17], and neoantigens $[18,19]$, in pre- and post-CCRT samples [20-22]. We also interpreted the clinical implications of these immunogenomic changes based on surgical pathology, immune cell fractions calculated by CIBERSORT [23] and immune scores calculated by ESTIMATE [24]. At the same time, we conducted multiplex immunohistochemistry (IHC) in representative paired samples.

\section{Methods}

\section{Study flow and patients}

Patients with esophageal cancer at clinical stages T1b-T4a and $\mathrm{NO}$ or $\mathrm{N}+$ were evaluated for surgery by a multidisciplinary team that included a medical oncologist, a radiation oncologist, a radiologist, and a thoracic surgeon. The surgical candidates $(n=40)$ underwent neoadjuvant chemoradiotherapy. Radiation therapy was delivered with a dose of
$4300-4400$ cGy in daily $210-215$ cGy per fraction over 4 to 5 weeks. Concurrent chemotherapy of 5 -FU $\left(4000 \mathrm{mg} / \mathrm{m}^{2}\right.$ over 4 days) and cisplatin $\left(60 \mathrm{mg} / \mathrm{m}^{2}\right.$ on day 1$)$ were administered every 3 weeks for up to two cycles during RT. Endoscopic biopsies were performed at the time of initial diagnosis and 2 to 3 weeks after the start of preoperative CCRT (Fig. 1a). This genomic analysis study was approved by the institutional review board of the Samsung Medical Center (IRB no. SMC-2013-10-112) and written informed consent was obtained from all enrolled patients.

\section{Isolation of genomic DNA and RNA}

Genomic DNA (gDNA) and RNA were purified from cancer tissues using the AllPrep DNA/RNA Mini Kit (Qiagen, USA). gDNA from peripheral blood was extracted using the QIAamp DNA Blood Mini Kit (Qiagen). gDNA concentration and purity were measured using a NanoDrop 8000 UV-Vis Spectrometer (Thermo Scientific Inc., USA) and a Qubit 2.0 Fluorometer (Life Technologies Inc., USA). To measure DNA degradation, median DNA size and $\Delta \mathrm{Ct}$ values were measured using a 2200 TapeStation Instrument (Agilent Technologies, USA) and real-time polymerase chain reaction (PCR; Agilent Technologies), respectively. RNA concentration and purity were measured using the NanoDrop and Bioanalyzer (Agilent Technologies).

\section{Whole-exome sequencing}

High quality gDNA in each sample was sheared with an S220 ultra-sonicator (Covaris, USA) and used to construct a library with the SureSelect XT Human All Exon v5 and SureSelect XT reagent kit, HSQ (Agilent Technologies), according to the manufacturer's protocol. This kit is designed to enrich 335,756 exons from 21,058 genes, covering $\sim 71$ $\mathrm{Mb}$ of the human genome. Enriched exome libraries were multiplexed and sequenced on the HiSeq 2500 platform (Illumina, USA). A paired-end DNA sequencing library was prepared via gDNA shearing, end-repair, A-tailing, paired-end adaptor ligation, and amplification. After hybridizing the library with bait sequences for $16 \mathrm{~h}$, the captured library was purified and amplified with an indexing barcode tag, and library quality and quantity were assessed using a 2200 TapeStation Instrument and Qubit 2.0 Fluorometer, respectively. The exome library was sequenced using the 10-bp paired-end mode of the TruSeq Rapid PE Cluster kit and the TruSeq Rapid SBS kit (Illumina).

\section{Exome sequence data analysis}

The University of California Santa Cruz hg19 reference genome (downloaded from http://genome.ucsc.edu) was used to align sequencing reads via the Burrows-Wheeler Aligner v. 0.6.2(15) with default settings. PCR duplications 


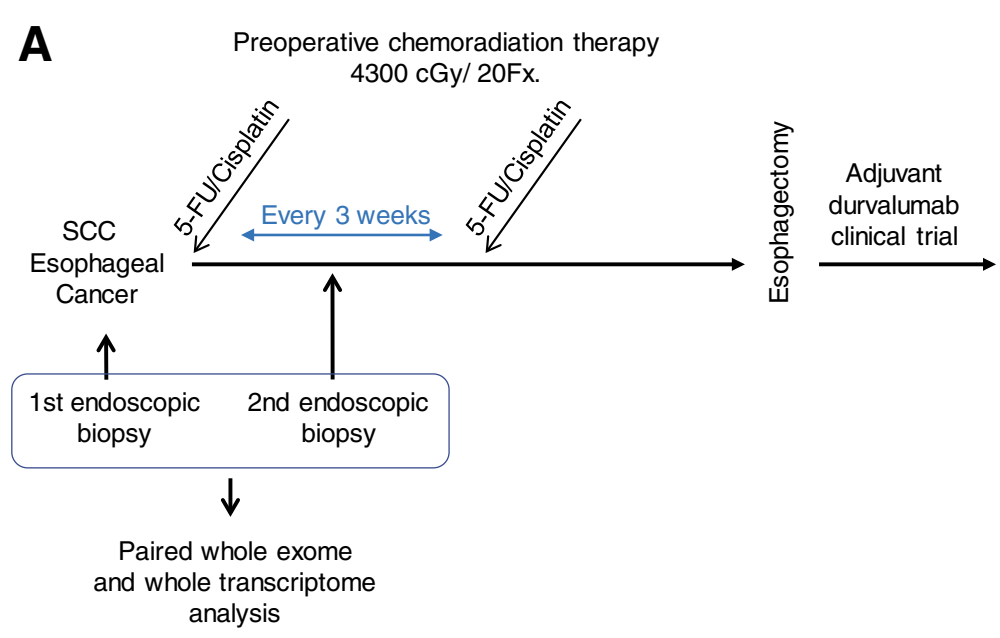

B

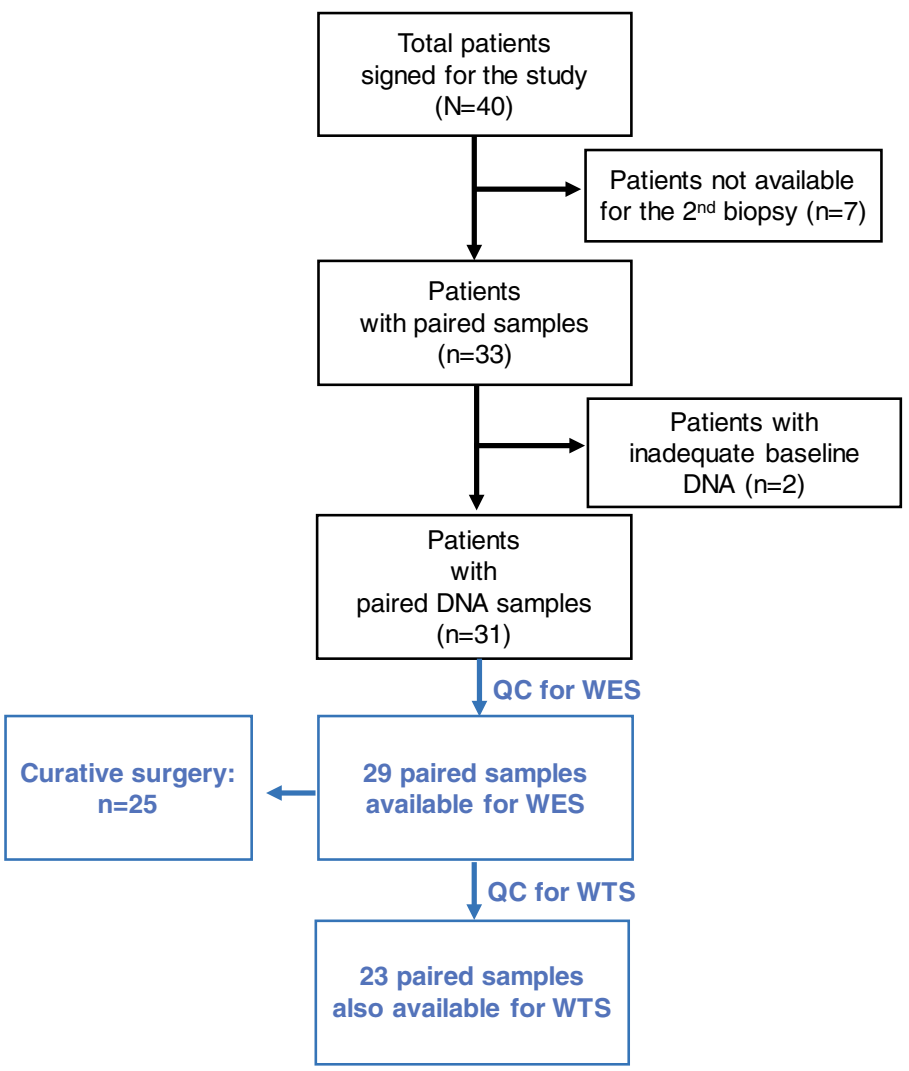

Fig. 1 Study flow and CONSORT diagram of the study population. a The first endoscopic sample was obtained at the time of diagnosis. The second sample was acquired at 2 to 3 weeks after initiation of concurrent chemoradiation therapy. b. Samples were acquired from 40 patients with signed informed consent and prepared for genomic analyses. Twenty-nine patients were available for the paired whole-exome sequencing (WES), and 23 patients were available for the paired whole-transcriptome sequencing (WTS). A total of 25 patients underwent curative surgery. Abbreviations: SCC, squamous cell carcinoma; QC, quality control

were marked using Picard-tools-1.8 (http://broadinstitute. github.io/picard/) and data cleanup was achieved using GATK-2.2.9 [25]. Point mutations were identified with the MuTect tool (https://github.com/broadinstitute/mutect) in paired samples. Indels were detected by VarScan2 (http://varscan.sourceforge.net/). Annovar was used to annotate variants. Signature analysis of mutational processes was carried out using the deconstructSigs tool [26]. Copy number variations were identified by EXCAVATOR [27]. Significant focal somatic copy number alterations were summarized by GISTIC analysis [28]. Tumor purity was estimated by $\mathrm{PyLOH}[29]$. 


\section{RNA sequencing}

Library construction for RNA sequencing (RNA-seq) was performed using a Truseq RNA Sample Preparation v2 Kit (Illumina). Isolated total RNA was used in a reverse transcription reaction with poly (dT) primers, using SuperScriptTM II Reverse Transcriptase (Invitrogen/Life Technologies, USA), according to the manufacturer's protocol. An RNA-seq library was prepared via cDNA amplification, end-repair, 3' end adenylation, adapter ligation, and amplification. Library quality and quantity were measured using the Bioanalyzer and Qubit. Sequencing of the RNA library was carried out using the 100-bp paired-end mode of the TruSeq Rapid PE Cluster Kit and the TruSeq Rapid SBS Kit (Illumina).

\section{RNA-Seq data analysis}

The reads from the FASTQ files were mapped against the hg19 human reference genome using TopHat version 2.0.6 (http://ccb.jhu.edu/software/tophat/index.shtml). Raw read counts mapped to genes were measured using the BAM format file by HTSeq version 0.6. 1 [30]. A total of 18,161 coding genes were analyzed for transcript abundance and poorly expressed genes were eliminated based on the criteria of a maximum read count $>20$ for all samples. Read counts were normalized using the Trimmed Mean of M-values normalization method. Differentially expressed genes were identified using the DESeq R package (www.huber.embl.de/users/anders/DESeq/). A gene set enrichment analysis (GSEA) [31] was conducted to analyze up- or down-regulated gene sets in specific groups of ESCC samples. Stromal and immune scores based on WTS were calculated using ESTIMATE [24]. Fractions of immune-associated cell types were calculated by CIBERSORT using RNA-seq expression profiles [23]. The immune cytolytic activity (CYT) was measured by the geometric mean of GZMA and PRF1 expression values in TPM [13].

\section{Cancer cell fraction measurement}

Cancer cell fractions (CCF) were measured by PyClone, which de-convolves tumor sequences into sub-clones based on a hierarchical Bayesian clustering model [32]. Input data were generated from somatic single-nucleotide variants (SNVs) detected by MuTect and corresponding copy number variations. SNVs were grouped into clusters with similar CCF values.

\section{Tumor mutation burden and prediction of candidate neoantigens}

TMB was measured by the number of somatic single nucleotide variants and indel mutations per megabase in the coding region [33]. Somatic single nucleotide variants include nonsynonymous as well as synonymous mutations. Non-coding alterations were not counted. In addition, known somatic alterations in COSMIC and truncations in tumor suppressor genes were excluded from the count.

Neoantigens were predicted using MuPeXI v.1.1.3 [34]. The three types of human leukocyte alleles (HLA-A, -B, and $-C$ ) were identified from the WTS data of each patient using seq2HLA [35]. Somatic mutations, gene expression counts, HLA types for each patient, and peptide lengths (811 mer) were provided as input for MuPeXI. Peptides with a half maximal inhibitory concentration (IC50) value $\leq 500$ $\mathrm{nM}$ were considered to have a high binding affinity for the major histocompatibility complex (MHC). Expressed mutant peptide sequences with an IC50 value of $\leq 500 \mathrm{nM}$ that showed binding affinity above normal were picked as candidate neoantigens.

\section{Multiplex immunohistochemistry (IHC) and analysis}

4- $\mu \mathrm{m}$ sections of specimens were cut from formalin-fixed paraffin-embedded (FFPE) blocks. Slides were heated for at least one hour in a dry oven at $60^{\circ} \mathrm{C}$ and dewaxed using xylene, then followed by multiplex immunofluorescence staining with a Leica Bond $\mathrm{Rx}^{\mathrm{Tm}}$ Automated Stainer (Leica Biosystems, Newcastle, UK). Briefly, the slides were baked for $30 \mathrm{~min}$ and dewaxed with Leica Bond Dewax solution (\#AR9222, Leica Biosystems), followed by antigen retrieval with Bond Epitope Retrieval 2 (\#AR9640, Leica Biosystems) in a $\mathrm{pH} 9.0$ solution for $30 \mathrm{~min}$. The first primary antibodies for CD4 (ab133616, Abcam, dilution 1:100) were incubated for $30 \mathrm{~min}$, followed by detection using the Polymer HRP Ms. + Rb (ARH1001EA, Perkin-Elmer) for $10 \mathrm{~min}$. Visualization of CD4 was accomplished using Opal 570 TSA Plus (dilution 1:150) for $10 \mathrm{~min}$, after which the slide was treated Bond Epitope Retrieval 1 (\#AR9961, Leica Biosystems) for $20 \mathrm{~min}$ to remove bound antibodies before the next step in the sequence. In a serial fashion, CD20 (ab9475, Abcam, ARH1001EA, Perkin-Elmer, Opal 520 TSA Plus), FOXP3 (ab20034, Abcam, ARH1001EA, Perkin-Elmer, Opal 690 TSA Plus), PD-L1 (13684S, Cell Signaling, ARH1001EA, Perkin-Elmer, Opal 620 TSA Plus), CD8 (MCA1817T, BIO-RAD, ARH1001EA, PerkinElmer, Opal 480 TSA Plus) and CK (M3515, Dako, ARH1001EA, Perkin-Elmer, Opal 780 TSA Plus) was stained. Nuclei were subsequently visualized with DAPI, and the section was coverslipped using HIGHDEF ${ }^{\circ}$ IHC fluoromount (ADI-950-260-0025, Enzo).

Slides were scanned using the PerkinElmer Vectra 3.0 Automated Quantitative Pathology Imaging System (Perkin-Elmer, Waltham, MA), and images were analyzed using the inform 2.2 software and TIBCO Spotfire ${ }^{\text {ma }}$ (Perkin-Elmer, Waltham, MA). Each cell was identified by detecting nuclear spectral elements (DAPI). All the immune cell populations from each panel were characterized and quantified using the cell segmentation tool by the InForm image analysis software. All cells in each slide were designated as 
positive or negative for each antibody, and the data were categorized and exported to an xls file for analysis. We used the Spotfire ${ }^{\mathrm{Tx}}$ program after tissue and cell segmentation and expression intensity was compared and then judged based on the cut-off value. The numbers of CD4, CD20, FOXP3, PD-L1, CD8, and CK positive cells were counted in each slide.

\section{Statistics}

The two-sided t-test was used for comparisons of tumor purity, mutation burden, immune and stromal score, cytolytic score, fraction of immune cell from the pre- and postCCRT samples. The Kaplan-Meier curves and log-rank test was used for the survival analysis. $P$-value less than 0.05 was considered as a statistically significant.

\section{Results}

\section{Baseline demographics}

From a total of 40 study participants, 29 and 23 patients participated in paired DNA analysis and whole-transcriptome analysis, respectively (Fig. 1b). The pre-treatment clinical stages were IVA $(n=4)$, III $(n=21)$, and II $(n=4)$. Of the 29 patients that enrolled for paired genomic analysis, four patients did not undergo surgery due to disease progression $(n=2)$, refusal of surgery, and failure to follow-up. Surgical samples after CCRT $(n=25)$ showed post-neoadjuvant stages of IVA $(n=2)$, IIIB $(n=3)$, IIIA $(n=7)$, II $(n=1)$, I $(n=7)$ and 5 cases of pathologic complete response (pCR). The first biopsy was conducted at a median time of 4 days (range 1-14) before CCRT and the second biopsy was conducted at a median of 18 days (range 4-24) after CCRT. The resection margins were negative for all samples. Seventeen patients are currently enrolled in other clinical trial, which aims to study the effects of randomized adjuvant durvalumab treatment versus placebo (NCT02520453; Additional file 1: Table S1) [36].

\section{Changes in the somatic mutation landscape and copy number alteration in pre- and post-CCRT samples}

We compared the somatic mutation landscape between pre- and post-CCRT samples, and analyzed representative genes related to cell cycle, histone modification, Hippo pathway, Notch pathway, and the PIK3CA pathway (Fig. 2a). Twenty-four pre-CCRT samples had missense, nonsense, or splicing mutations in the tumor suppressor gene, TP53, and these alterations were maintained in 11 samples after CCRT. Although nine pre-CCRT samples had a missense mutation in the nuclear factor erythroid 2 like 2 gene, NFE2L2, only two post-CCRT samples retained that mutation. SNV analysis showed that transition mutations, specifically $\mathrm{C}$ to $\mathrm{T}$ or $\mathrm{G}$ to $\mathrm{A}$, were prominent in all samples. Comparison of copy number amplification and deletion regions between genomes of pre-CCRT and post-CCRT samples (Fig. 2b), showed that copy number at chromosome 7p14.1 was amplified only in post-CCRT samples. Additionally, the region harboring the cyclin D1 (CCND1) oncogene was amplified and the region harboring the tumor suppressor genes, Cyclin Dependent Kinase Inhibitor 2A (CDKN2A) and CDKN2B, was deleted in both pre- and post-CCRT samples.

Analysis of TMB, immune and stromal score profile, and immune cell composition in the tumor microenvironment in pre- and post-CCRT samples

We found no difference in tumor purity, between pre- and post-CCRT samples (Fig. 2c). This allowed us to analyze the TMB and neoantigen load using WES and WTS data. We found that the TMB and neoantigen load were significantly lower in post-CCRT samples $(p<0.001)$ compared with pre-CCRT samples (Fig. 2d). Using ESTIMATE (Estimation of STromal and Immune cells in MAlignant Tumor tissues using Expression data), we found that 14 post-CCRT samples (60.9\%) showed an increased immune score compared with pre-CCRT samples. Of those 14 post-CCRT samples, 10 also showed a concurrent increase in the stromal score (Fig. 3a). Therefore, CCRT leads to increased immune and stromal scores in the tumor tissue (Figs. 3b and c). No difference in immune CYT was observed between pre- and post-CCRT samples (Fig. 3d). Using CIBERSORT, we analyzed the changes in the population of immune cell types before and after CCRT. We found that the numbers of resting natural killer $(\mathrm{NK}) / \mathrm{T}$-cells $(p<0.001)$, follicular helper T-cells $(p=0.049)$, and regulatory T-cells $(p=0.038)$ significantly decreased after CCRT. However, the population of neutrophils was significantly increased in the post-CCRT samples $(p=0.001$; Fig. 3e). We found no difference in the abundance of major immune cells such as B-cells, CD4 T-cells, CD8 T-cells, and M0, M1, and M2 macrophages between pre- and post-CCRT cells.

Multiplex immunohistochemistry results in pre- and postCCRT samples

We found a strong correlation between outcomes from gene expression profile and multiplex IHC. Multiplex IHC for CD8 was significantly correlated with CYT score ( $p=$ 0.012). Especially PRF1 (Perforin 1) expression among CYT genes was significant $(p=0.001)$ while GRZA (Granzyme A) was not significant $(p=0.105)$. IFN-r was also correlated with CD8 $(p=0.104)$, and PD-L1 expression was negatively correlated with CD8 $(p=0.022)$ (Fig. 4a). Multiplex IHC is conducted in four patients available for both pre- and post- CCRT samples (Fig. 4b, Additional file 2: Table S2). Interestingly, all the samples showed an incremental trend in CD4 cell proportion after the CCRT. The proportion of cell expressing CD8, CD20, FOXP3, PD-L1 showed no definitive trend. (Fig. 4c). TMB was also highly correlated with CD8, but it was not significant $(r=0.69$, $p=0.059$ ) (data not shown). 


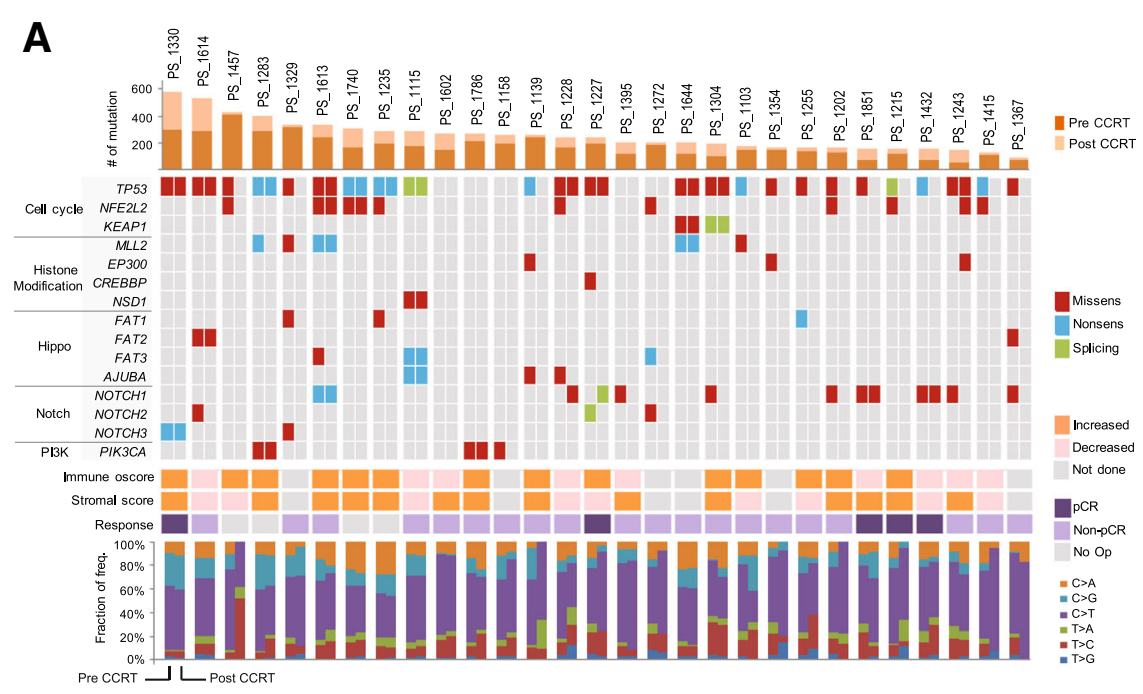

B
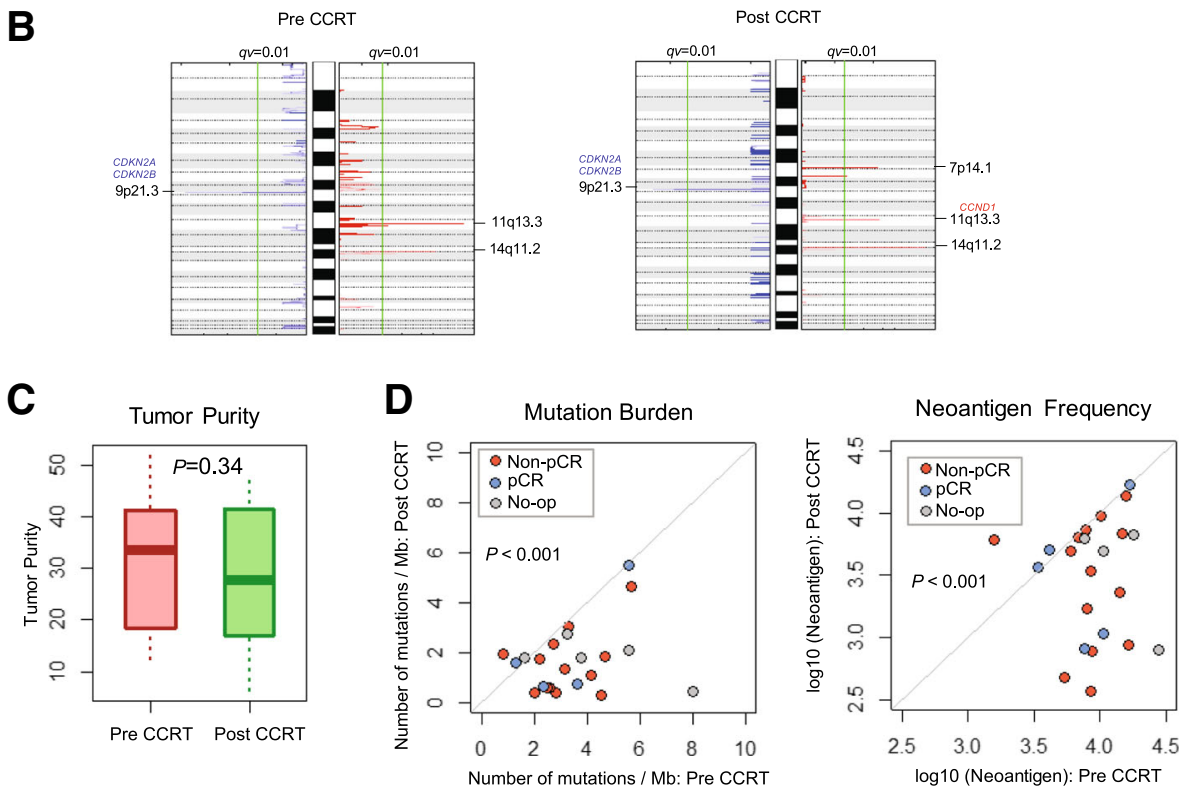

Fig. 2 Tumor mutation profile and changes in copy number, mutation burden in pre- CCRT and post-CCRT biopsy samples. a, Heatmap of somatic mutations (including missense, nonsense, and splicing) detected in 29 study patients. Each patient had a paired column (1st: Pre-CCRT and 2nd: Post-CCRT). Samples were annotated for mutation quantity, immune or stromal score changes, and surgical pathology. Each bar portrayed in the bottom represents the proportion of each type of substitution. $\mathbf{b}$, Copy number alteration was analyzed from pre- and post-samples. c, Tumor purity was calculated by whole-exome sequencing. $\mathbf{d}$, Comparison of mutation burden and neoantigen frequency between pre-CCRT and post-CCRT samples $(n=29)$. Abbreviations: CCRT, concurrent chemoradiation therapy; pCR, pathologic complete response

Tissue characteristics and survival analysis of patients with an increased immune score after CCRT

The 14 post-CCRT samples that showed increased immune scores also showed an increased abundance of neutrophils and a decrease in the number of follicular helper T-cells, regulatory $\mathrm{T}$-cells, and resting NK/T-cells compared with the baseline pre-CCRT samples. We found that pre-CCRT samples with a higher proportion of M0 macrophages and lower resting mast cells were likely to show an increase in immune score after CCRT (Additional file 3: Table S3).
Survival analysis showed extended PFS and overall survival (OS) in patient subgroups with increased immune scores (12-month PFS rate: $83.1 \%$ vs. $57.1 \%, p=0.248$; 12 -month OS rate: $92.3 \%$ vs. $85.7 \%, p=0.702$ ) compared with the remaining subgroups (Fig. $5 \mathrm{a}$ and $\mathrm{b}$ ). To validate the strength of these potential prognostic factors, we analyzed patient survival using $\mathrm{pCR}$, a well-known prognostic factor for ESCC [37]. Disease free survival (DFS) and OS were extended in patients that showed pCR after CCRT (12-month DFS rate: $100.0 \%$ vs. $62.2 \%, p=0.210 ; 12$-month OS rate: 
A

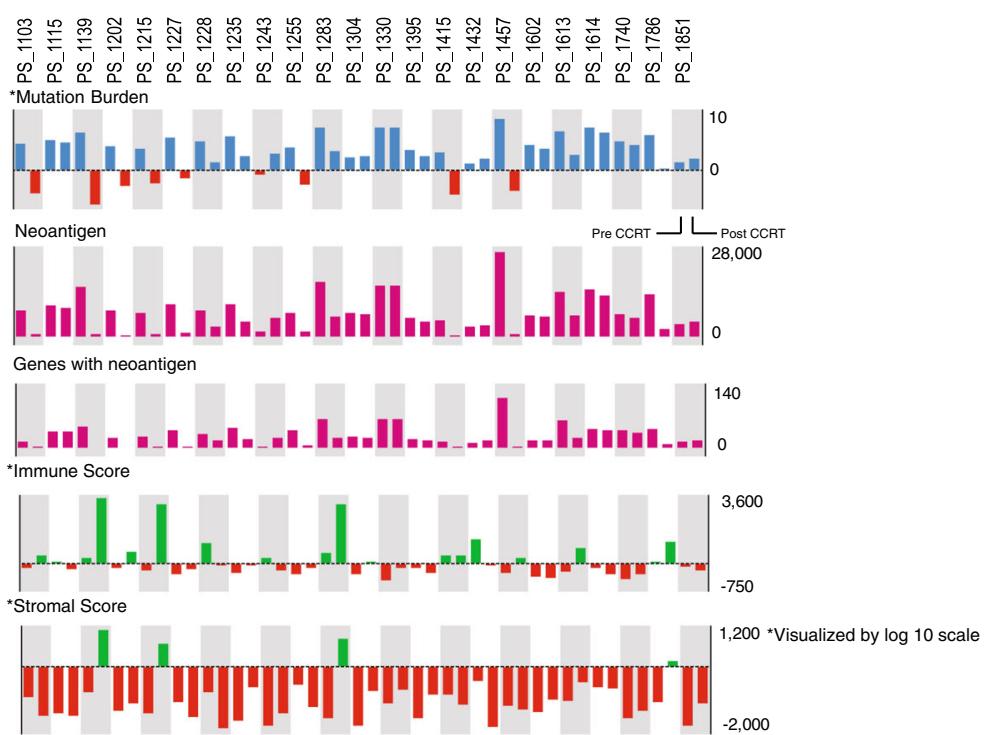

B

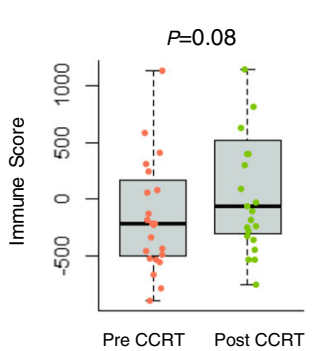

C

E

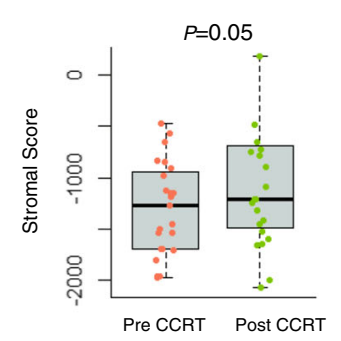

D
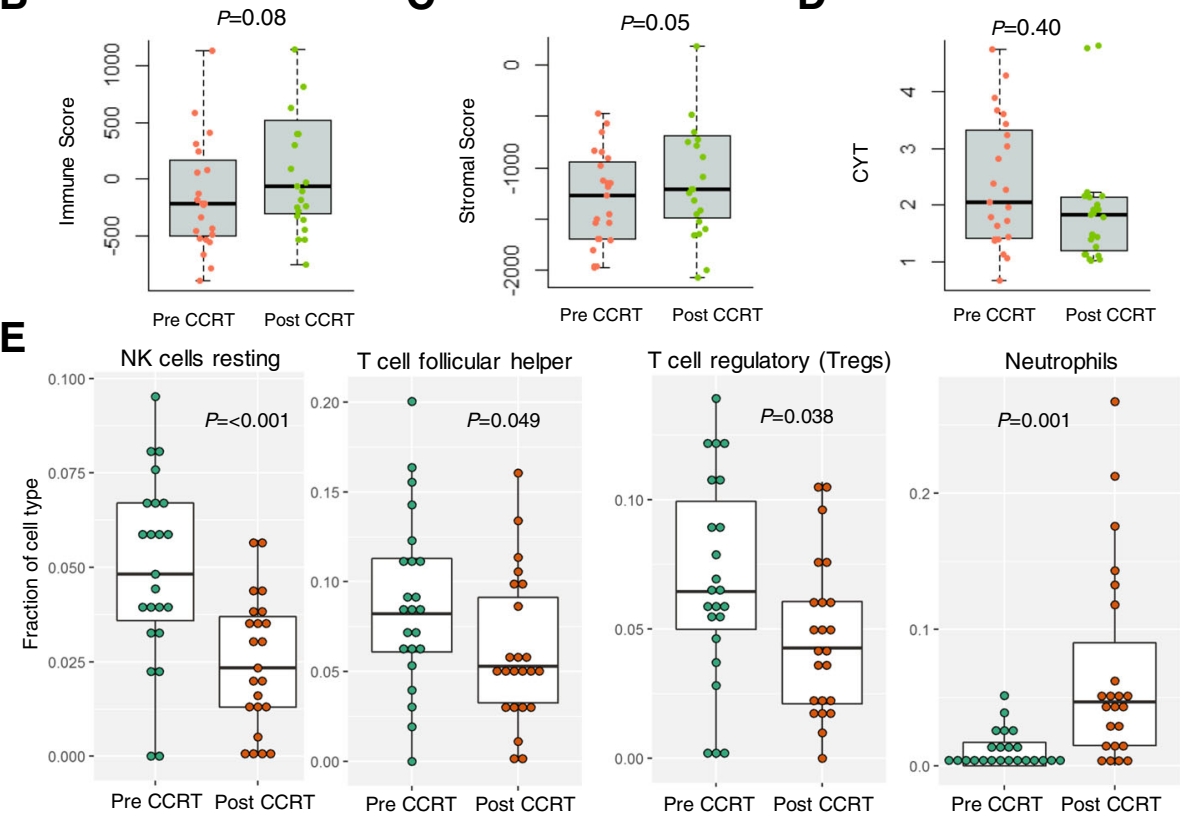

Fig. 3 Comparison of changes in immune cell fractions, and immune, stromal, and immune cytolytic activity scores between pre-CCRT and postCCRT samples. a Details regarding the change in each sample based on pre-CCRT and post-CCRT samples. Comparison of the immune and stromal score (b) and (c), and immune cytolytic (CYT) activity (d), in samples available for paired whole-transcriptome sequencing $(n=23) . \mathbf{e}$, The fraction of immune cells showing statistically significant changes $(n=23)$. Abbreviations: CCRT, concurrent chemoradiation therapy, CYT, cytolytic activity

$100.0 \%$ vs. $82.2 \%, p=0.465)$, which was similar to the difference in survival rates analyzed according to the change of immune score (Figs. 5c and d).

\section{Differentially expressed gene analysis and gene set enrichment analysis}

Sixty genes that satisfied pre-defined criteria $(>2$-fold change and adjusted $p<0.01)$ were identified by differentially expressed gene analysis in pre- and post-CCRT tissue samples (Fig. 6a and Additional file 4: Table S4). We found that the expression of cell cycle-related tumor suppressor genes, CCND2 and CDKN1A, was increased after CCRT. Gene set enrichment analysis showed that several immune-related gene sets, such as those involved in interferon gamma signaling, cytokine signaling, adaptive immune system, innate immune system, PD1 signaling, T-cell receptor signaling, and CD28 co-stimulation were enriched in post-CCRT samples (Figs. $6 \mathrm{~b}$ and c). 
A
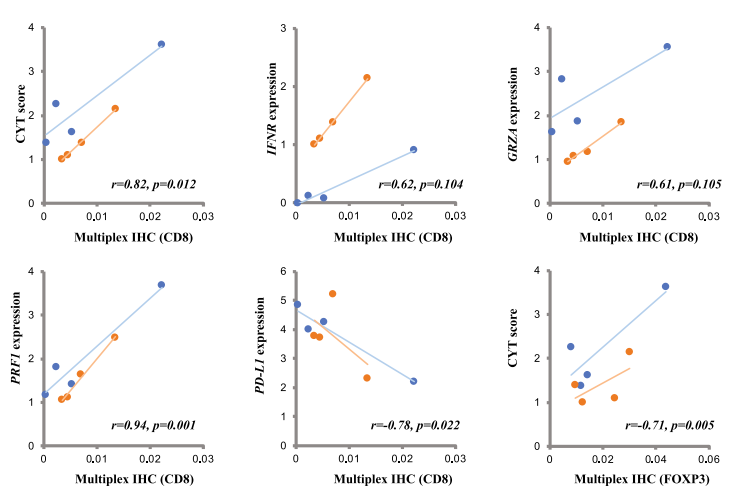

- Pre-CCRT Post-CCRT

B

Pre-CCRT

(PS-1740 Sample)

Post-CCRT
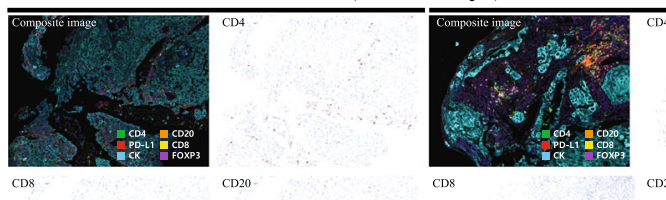

$\operatorname{CD} 20$

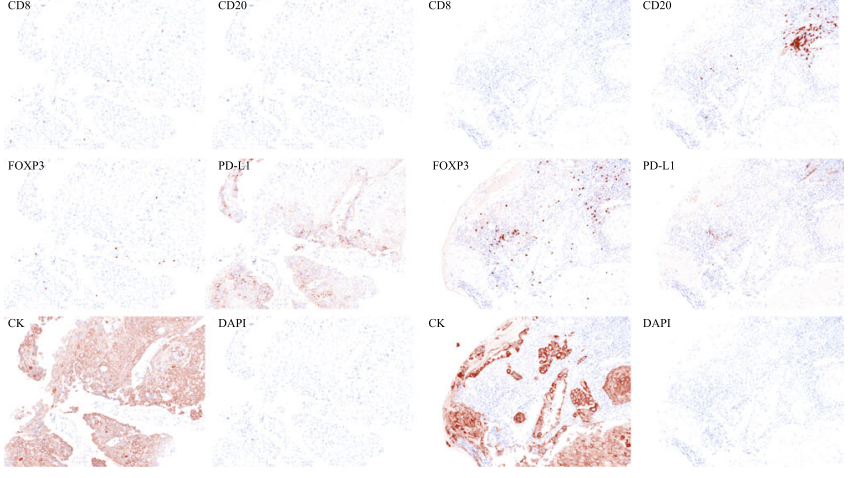

C
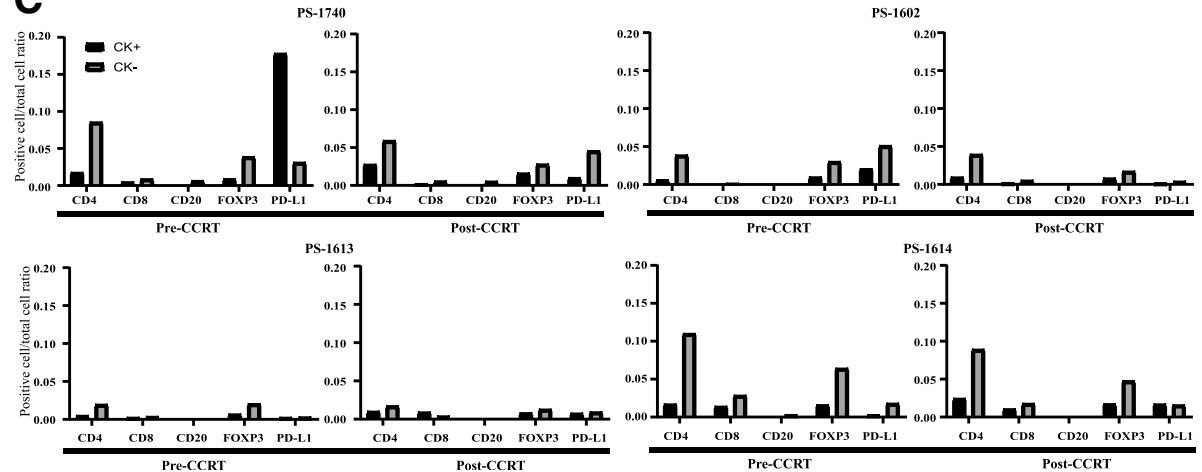

Fig. 4 a Correlation between multiplex IHC result and expression of genes and gene sets. CYT score, PERF1, PD-L1 were correlated with CD8 positive cells and CYT was correlated with FOXP3 positive cell by multiplex IHC. Pearson's $r$ and $P$-value is calculated using both pre- and postCCRT sample. b Represent images from multiplex IHC (PS-1740) showing result from pre-CCRT and post-CCRT samples. c The proportion of cells per total cell in shown based on cytokeratin positivity

\section{Correlation of genomic profile with treatment outcome}

To analyze the genomic profile of the ESCC tissue samples based on their surgical pathology, we compared pCR ( $n=$ $5)$ and non-pCR $(n=20)$ subgroups using pre- andpostCCRT samples. We observed no significant difference in immune and stromal scores between the two subgroups in pre-CCRT samples. However, the pCR subgroup showed significantly lower immune CYT compared with the
non-pCR subgroup $(p=0.011)$ in pre-CCRT samples. We found a prominent decrease in CYT in the non-pCR group $(p=0.047)$ after CCRT, but no change in CYT in the pCR group compared with pre-CCRT samples (Fig. 7a). We also did not find any predictive somatic mutation markers in the pCR subgroup. NFE2L2, a gene frequently found to be mutated in our pre-CCRT samples, mostly disappeared after CCRT (Fig. 3a). CCF estimation analysis showed that 


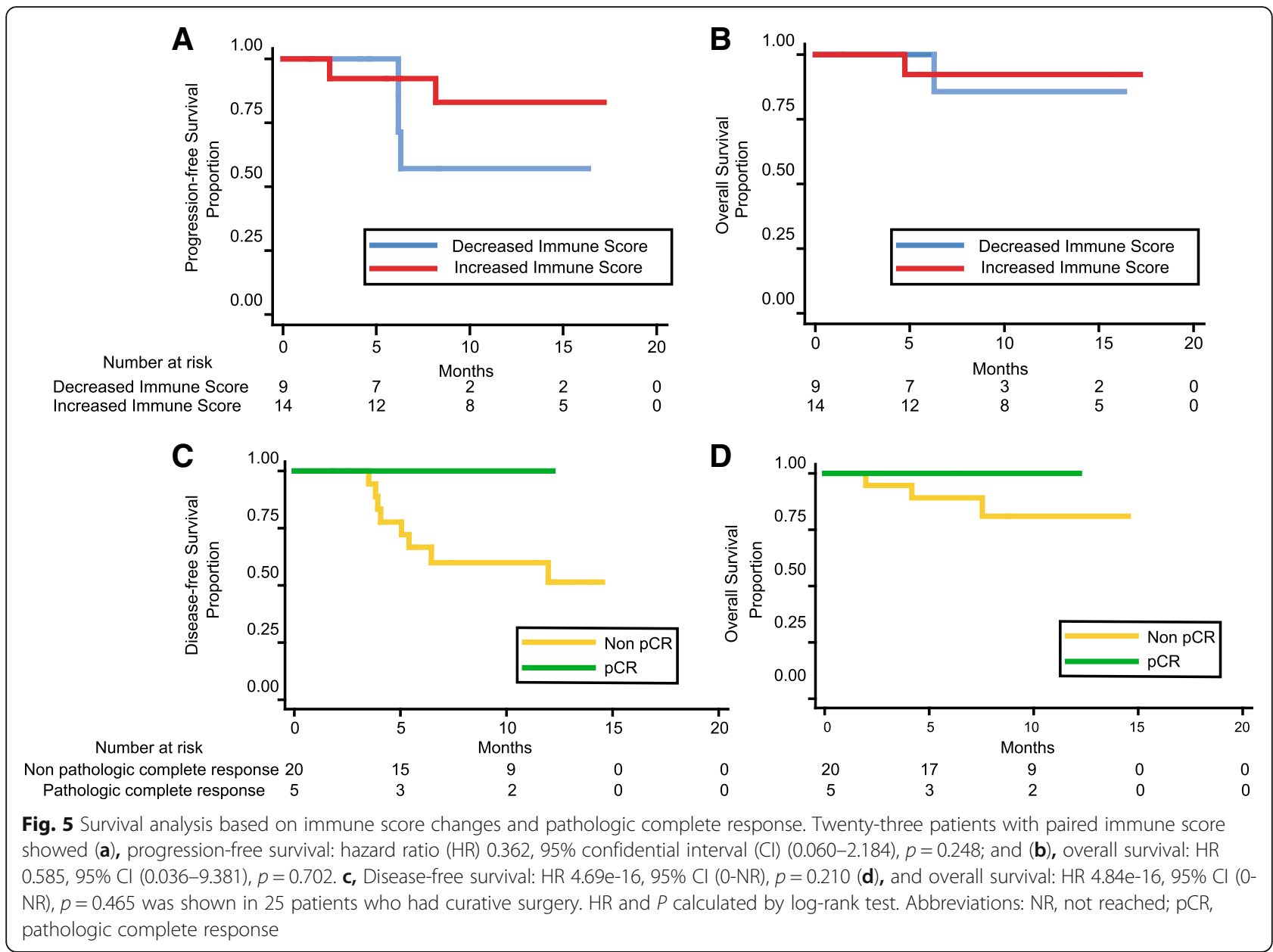

NFE2L2 p.D15E was the only unique variant in pCR samples. However, the proportion of cancer cells with specific mutation failed to specifically correlate with the pathologic response (Fig. 7b). Analysis of changes in the population of immune cell types showed a significant increase in activated dendritic cells after CCRT in the $\mathrm{pCR}$ subgroup compared with pre-CCRT samples. Neutrophils were significantly increased in number in both $\mathrm{pCR}$ and non-pCR subgroups after CCRT compared with pre-CCRT samples (Fig. 7c and Additional file 5: Table S5). However, analysis of individual gene expression patterns revealed no specific pattern in the $\mathrm{pCR}$ and non-pCR subgroups before and after CCRT (Additional file 6: Table S6 and Additional file 7: Fig. S1).

In addition, we looked into the difference in immune profiles between samples from inoperable patients $(n=$ $2)$ and the patients who received surgery $(n=25)$. In pre-CCRT samples from inoperable patients showed significantly lower stromal and CYT scores compared with patients who received surgery $(P=0.0014$ and $P=0.012$, respectively). A similar pattern in immune scores was shown despite not significant (Additional file 8: Fig. S2). Looking into the immune cell compositions between two groups, a fraction of resting NK cells and regulatory $\mathrm{T}$ cells in pre-CCRT samples were significantly higher in inoperable patients compared to the patients who conducted surgery $(P=0.037$ and $P=0.005$, respectively). There was no significant difference in TMB and neoantigen load between both groups.

\section{Discussion}

Recent advances in modern sequencing and analytical tool-kits have resulted in the evolution of immunogenomics as a critical component of cancer immunotherapy. Immunogenomic studies help to understand the mechanisms that control therapy response and resistance to immune response shaped by the tumor and its microenvironment. In this study, we have comprehensively analyzed the changes in the immunogenomic profile of ESCC in response to CCRT.

Using our sequencing dataset, we analyzed the genomic profiles of patients with ESCC to identify subgroups that could benefit from CCRT. We found that patient subgroups with increased immune scores after CCRT showed favorable survival outcomes. However, the elevated immune score could be primarily due to an 

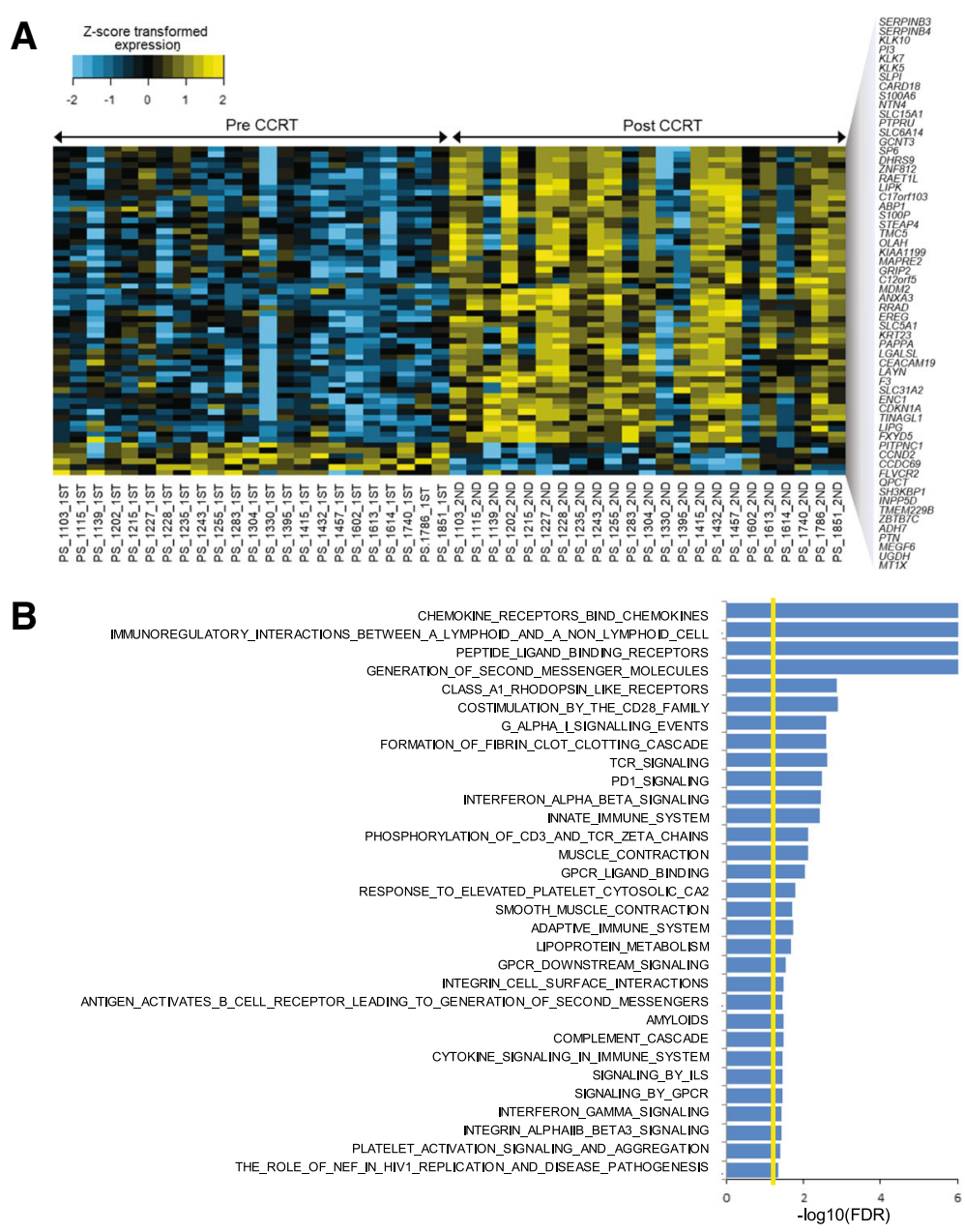

C

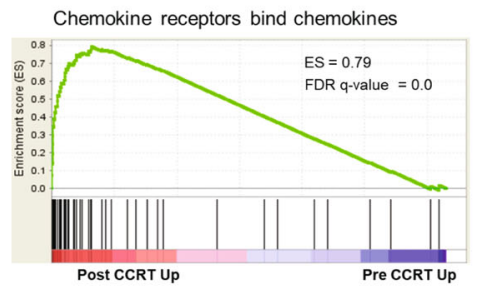

Costimulation by the $\mathrm{CD} 28$ family

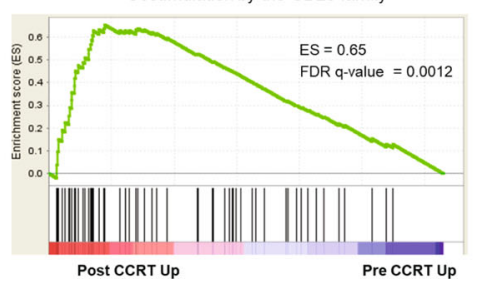

Fig. 6 Differentially expressed genes and gene set enrichment analysis in pre- and post-CCRT samples. a, Heatmap comparison of 60 significant genes ( $>2$-fold change \& adjusted $p<0.01$ ) between pre- and post-CCRT samples. $\mathbf{b}$, Enriched differentially expressed gene sets between pre-CCRT and post-CCRT. $\mathbf{c}$, Representative gene set enrichment analysis of differentially expressed genes in pre- and post-CCRT samples

increase in the abundance of neutrophils, not adaptive immune cells, in the tumor microenvironment. Therefore, our results suggested that neutrophils were the primary modulators of immungenomic changes during CCRT which also consequences comparably favorable survival outcomes. 

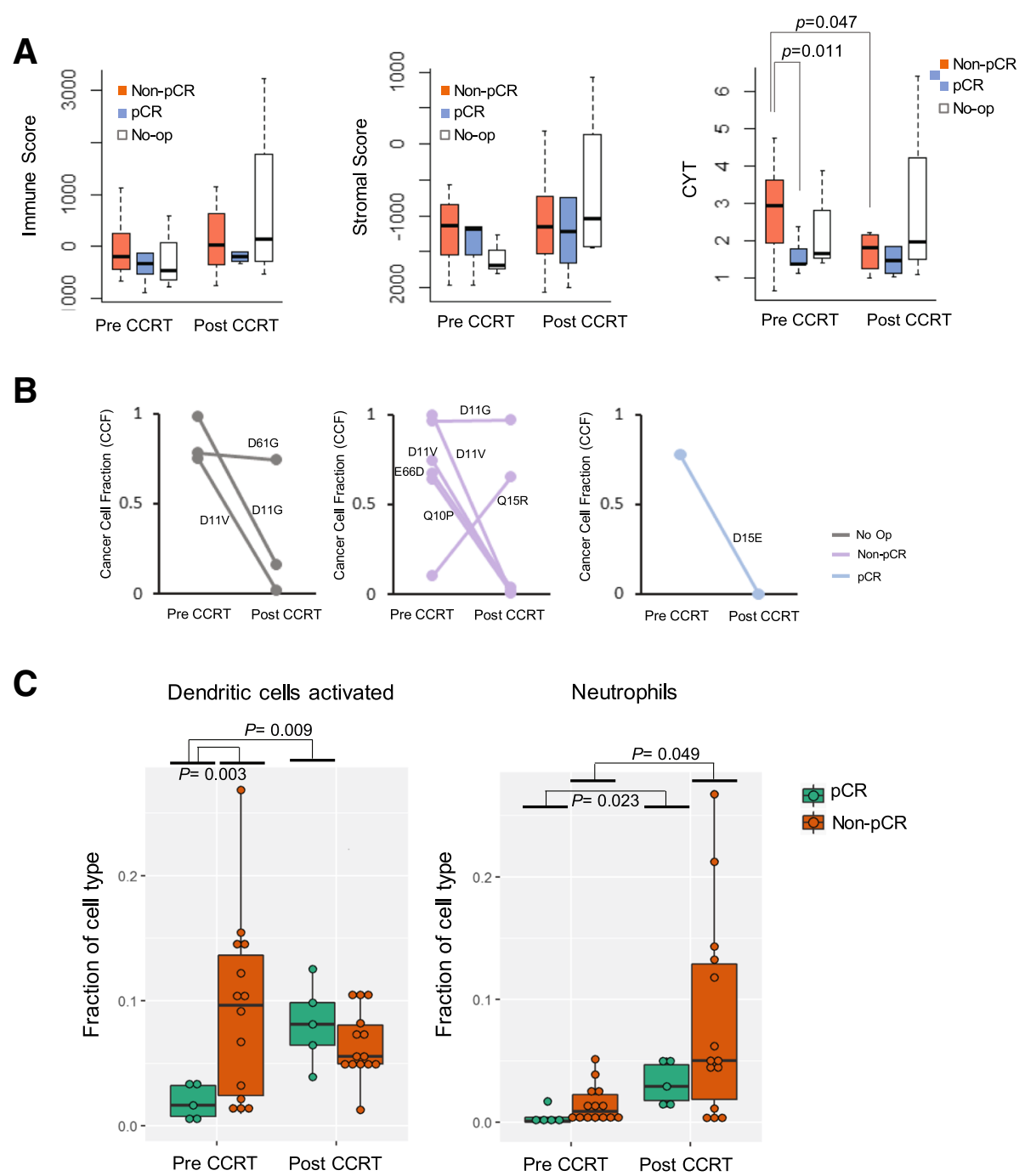

Fig. 7 Comparison of changes in the immune, stromal, and immune cytolytic activity scores and immune cell fractions between pre- CCRT and post-CCRT samples based on their surgical pathologic profile. a, Immune and stromal scores, and immune cytolytic (CYT) activity in pathologic complete response (pCR), non-pCR, and no-operations (Op) samples. b. Somatic mutation changes in NFE2L2 using cancer cell fraction measurements based on the surgical pathologic profile.c, Changes in immune cell fractions based on the surgical pathologic profile

Previous studies [38-40] have suggested that the molecular signature of NFE2L2 (NRF2), a master transcriptional regulator of stress response, serves as a predictive marker for esophageal tumor response to CCRT. A gain-of-function NRF2 mutation confers resistance to therapy in ESCC cells [39]. However, our results showed no significant correlation between NFE2L2 missense mutations and the pCR of the study population (pCR rate of $14.3 \%$ in patients with NFE2L2 mutations vs. $20.0 \%$ in the remaining patients). It is possible that the missense mutations observed in our study population failed to affect NFE2L2 function and further analysis is needed to study the functional alteration of the mutated forms of NFE2L2 in the ESCC tissue samples.
The tumor microenvironment contains diverse immune cell types in addition to tumors cells and its nature and composition change over time with treatment [41, 42]. From our previous study, it is known that PD-L1 expression elevated in ESCC samples that received preoperative CCRT compared to the CCRT naive sample [43]. These findings reflect that CCRT induces immune checkpoint protein expression in tumor which we could also expect alteration in immune cell composition in tumor microenvironment lead by upregulation of PD-L1. We found an enrichment of neutrophils in the tumor microenvironment after CCRT (Fig. 3e). However, we failed to see an increase in the number of adaptive immune cells needed for anti-tumor immune response, such as activated CD8 T-cells and dendritic cells, after CCRT. CYT, which is 
indicative of activated CD8 T-cells, was also not elevated after CCRT (Fig. 3d). Radiation is known to induce an inflammation response by damaging tumor endothelial cells and triggering inflammatory cytokine signaling (via Interleukin 1 and the tumor necrosis factor) [44, 45]. Radiation also induces the recruitment of circulating immune cells and increases antigen exposure to initiate an adaptive immune response [46]. Our results on neutrophil enrichment after CCRT suggested that chemoradiation, unlike radiation only, induce non-specific inflammation rather than an adaptive immune response in the tumor microenvironment of ESCC.

High TMB and neoantigen load in a tumor can generate T-cell responses that recognize and eradicate tumor cells [47]. Clinical trials have shown that high TMB increases the efficacy of immune checkpoint blockades in cancer immunotherapy [16]. We found that TMB and neoantigen load were significantly reduced $(p<0.001)$ in ESCC samples after CCRT. Radiotherapy is known to induce antigen presentation [46]. However, our results showed that underlying tumor mutation burden, which hypothetically shows positive correlation to tumor antigen presentation, were lowered after chemoradiation.

Based on our results that CCRT decreases the TMB and induces non-specific inflammation in ESCC cells, it is possible that the immune cell priming and reinvigoration induced by immune checkpoint inhibitors can have a greater impact when immunotherapy is combined before or at the time of initiating CCRT. Patients with non-small cell lung cancer (NSCLC) that undergo consolidation therapy with anti-PD-L1 inhibitor (durvalumab) following completion of CCRT show increased survival [7]. Concurrent durvalumab treatment with CCRT, starting with CCRT not after CCRT, is currently being tested for improved synergistic effect and survival benefit compared to previous result in same NSCLC population (NCT03519971) [48]. Also, current clinical trials are testing combination therapies in which immunotherapy is initiated one or two weeks prior to CCRT [49-51]. Results from these clinical trials may validate our hypothesis.

One of the challenges that we faced during this study was the selection of an optimal timepoint for the second biopsy to best identify the immunogenomic changes caused by CCRT. We had initially designed the study to compare tissue samples from patients with ESCC at the time of diagnosis and post-surgical samples from patients that had undergone CCRT. However, we were unable to perform genomic analyses on samples that were exposed to the entire CCRT regimen due to extensive tumor necrosis. Therefore, we conducted the second biopsy within 2 to 3 weeks after the initiation of CCRT. Due to the reason, small number of samples were available for the multiplex IHC which weakens the representative value of its result and showed inconsistent result in some result such as trend of CD4-cells before and after the CCRT. Future studies should confirm whether our timepoints were optimal for the evaluation of immunogenomic changes in the tumor and its microenvironment.

In conclusion, our study is the first to demonstrate the underlying genomic changes caused by CCRT. It will provide a basis for further genomic studies in patients that undergo CCRT and guide clinical trials that test combination therapy treatments of immunotherapy and CCRT.

\section{Additional files}

Additional file 1: Table S1. Clinical information of study population (XLS $2133 \mathrm{~kb}$ )

Additional file 2: Table S2. Samples available for the Multiplex $I H C$ (XLSX $11 \mathrm{~kb}$ )

Additional file 3: Table S3. The difference between groups for relative fractions of major immune cell types compared by increase in immune score calculated by ESTIMATE. The significance was measured by a t-test between two sample groups. (XLSX $13 \mathrm{~kb}$ )

Additional file 4: Table S4. Differentially expressed genes between preconcurrent chemoradiation therapy (CCRT) and post-CCRT (XLSX $1920 \mathrm{~kb}$ )

Additional file 5: Table S5. The difference between groups for relative fractions of major immune cell types compared by surgical pathology. The significance was measured by t-test between two sample groups. (XLSX $12 \mathrm{~kb}$ )

Additional file 6: Table S6. Differentially expressed genes between of pre-concurrent chemoradiation therapy (CCRT) and post-CCRT in no pCR and pCR patients (XLSX $3700 \mathrm{~kb}$ )

Additional file 7: Figure S1. Heatmap comparison of differentially expressed genes between pre- and post-concurrent chemoradiation in the non-pathologic complete response sample group. (PDF $499 \mathrm{~kb}$ )

Additional file 8: Figure S2. (a) comparison between samples from the patients who showed disease progression (PD) to neo-adjuvant chemotherapy $(n=2)$ and the samples from the patients (Other) who received surgery $(n=25)$. (b) Specific immune cells which showed significant higher fraction compared between the neoadjuvant chemotherapy PD patients and other patients (PDF $543 \mathrm{~kb}$ )

\section{Abbreviations}

5-FU: 5-fluorouracil; CCF: cancer cell fraction; CCRT: concurrent chemoradiation therapy; CYT: cytolytic activity; DFS: disease free survival; ESCC: esophageal squamous cell carcinoma; gDNA: genomic DNA; GSEA: gene set enrichminet analysis; HLA: human leukocyte alleles; IC: inhibitory concentration; MHC: major histocompatibility complex; OS: overall survival; PCR: polymerase charin reaction; PD-L1: programmed cell death-ligand 1; PFS: progression-free survival; RNA-seq: RNA sequencing; SCC: squamous cell carcinoma; SNVs: single nucleotide variants; TMB: tumor mutation burden; WES: whole-exome sequencing; WTS: whole-transcriptome sequencing

\section{Acknowledgements}

Not applicable.

Funding

This study was supported by the National R \& D Program for Cancer Control, Ministry of Health \& Welfare, Korea (1720180).

Availability of data and materials

The dataset used and/or analyzed during the current study is provided in supplementary materials and additional material are available from the corresponding author on reasonable request. 


\section{Authors' contributions}

Conception and design: JS, SP, JJ, YWM, Development of methodology: JS, SP, JJ, YWM, Acquisition of data: JS, YWM, DO, SHL, YLC, JSA, MJA, KP, Analysis and interpretation of data: JS, SP, JJ, YWM, JYN, DR, WYP, Administrative, technical, or material support: JS, SP, JJ, JN, DR, WYP, Study supervision: JS, SP, JJ, YWM, All authors read and approved the final manuscript

\section{Ethics approval and consent to participate}

This genomic analysis study was approved by the institutional review board of the Samsung Medical Center (IRB no. SMC-2013-10-112) and written informed consent was obtained from all enrolled patients.

\section{Consent for publication}

Not applicable.

\section{Competing interests}

The authors declare that they have no competing interests.

\section{Publisher's Note}

Springer Nature remains neutral with regard to jurisdictional claims in published maps and institutional affiliations.

\begin{abstract}
Author details
${ }^{1}$ Division of Hematology-Oncology, Department of Medicine, Samsung Medical Center, Sungkyunkwan University School of Medicine, 81 Irwon-ro, Gangnam-Gu, Seoul 60351, Republic of Korea. ${ }^{2}$ Samsung Genome Institute, Samsung Medical Center, Sungkyunkwan University School of Medicine, Seoul, Republic of Korea. ${ }^{3}$ Division of Gastroenterology, Department of Medicine, Samsung Medical Center, Sungkyunkwan University School of Medicine, Seoul, Republic of Korea. ${ }^{4}$ Department of Radiation Oncology, Samsung Medical Center, Sungkyunkwan University School of Medicine, Seoul, Republic of Korea. ${ }^{5}$ Department of Pathology and Translational Genomics, Samsung Medical Center, Sungkyunkwan University School of Medicine, Seoul, Republic of Korea.
\end{abstract}

\section{Received: 1 December 2018 Accepted: 2 May 2019}

Published online: 16 May 2019

\section{References}

1. Torre LA, Bray F, Siegel RL, Ferlay J, Lortet-Tieulent J, Jemal A. Global cancer statistics, 2012. CA Cancer J Clin. 2015;65:87-108.

2. Sjoquist KM, Burmeister BH, Smithers BM, Zalcberg JR, Simes RJ, Barbour A et al. Survival after neoadjuvant chemotherapy or chemoradiotherapy for resectable oesophageal carcinoma: an updated meta-analysis. Lancet Oncol. 2011;12:681-92.

3. van Hagen P, Hulshof MC, van Lanschot JJ, Steyerberg EW, van Berge Henegouwen MI, Wijnhoven BP, et al. Preoperative chemoradiotherapy for esophageal or junctional cancer. N Engl J Med. 2012;366:2074-84.

4. Swisher SG, Hofstetter W, Komaki R, Correa AM, Erasmus J, Lee JH, et al. Improved long-term outcome with chemoradiotherapy strategies in esophageal cancer. Ann Thorac Surg. 2010;90:892-8 discussion 8-9.

5. Pardoll DM. The blockade of immune checkpoints in cancer immunotherapy. Nat Rev Cancer. 2012;12:252-64.

6. Hargadon KM, Johnson CE, Williams CJ. Immune checkpoint blockade therapy for cancer: an overview of FDA-approved immune checkpoint inhibitors. Int Immunopharmacol. 2018;62:29-39.

7. Antonia SJ, Villegas A, Daniel D, Vicente D, Murakami S, Hui R, et al. Durvalumab after Chemoradiotherapy in stage III non-small-cell lung Cancer. N Engl J Med. 2017;377:1919-29.

8. Liu XS, Mardis ER. Applications of Immunogenomics to Cancer. Cell. 2017; 168:600-12.

9. Ock CY, Keam B, Kim S, Lee JS, Kim M, Kim TM, et al. Pan-Cancer Immunogenomic perspective on the tumor microenvironment based on PD-L1 and CD8 T-cell infiltration. Clin Cancer Res. 2016;22:2261-70.

10. Rizvi H, Sanchez-Vega F, La K, Chatila W, Jonsson P, Halpenny D, et al. Molecular determinants of response to anti-programmed cell death (PD)-1 and anti-programmed death-ligand 1 (PD-L1) blockade in patients with non-small-cell lung Cancer profiled with targeted next-generation sequencing. J Clin Oncol. 2018;36:633-41.
11. Zaretsky JM, Garcia-Diaz A, Shin DS, Escuin-Ordinas H, Hugo W, HuLieskovan S, et al. Mutations associated with acquired resistance to PD-1 blockade in melanoma. N Engl J Med. 2016;375:819-29.

12. Skoulidis F, Goldberg ME, Greenawalt DM, Hellmann MD, Awad MM, Gainor JF, et al. STK11/LKB1 mutations and PD-1 inhibitor resistance in KRASmutant lung adenocarcinoma. Cancer Discov. 2018;8:822-35.

13. Rooney MS, Shukla SA, Wu CJ, Getz G, Hacohen N. Molecular and genetic properties of tumors associated with local immune cytolytic activity. Cell. 2015;160:48-61.

14. Gao J, Shi LZ, Zhao H, Chen J, Xiong L, He Q, et al. Loss of IFN-gamma pathway genes in tumor cells as a mechanism of resistance to anti-CTLA-4 therapy. Cell. 2016;167:397-404 e9.

15. Hellmann MD, Nathanson T, Rizvi H, Creelan BC, Sanchez-Vega F, Ahuja A, et al. Genomic features of response to combination immunotherapy in patients with advanced non-small-cell lung Cancer. Cancer Cell. 2018;33: 843-52 e4.

16. Hellmann MD, Ciuleanu TE, Pluzanski A, Lee JS, Otterson GA, AudigierValette C, et al. Nivolumab plus Ipilimumab in lung Cancer with a high tumor mutational burden. N Engl J Med. 2018;378:2093-104.

17. Hugo W, Zaretsky JM, Sun L, Song C, Moreno BH, Hu-Lieskovan S, et al. Genomic and transcriptomic features of response to anti-PD-1 therapy in metastatic melanoma. Cell. 2016;165:35-44.

18. Rizvi NA, Hellmann MD, Snyder A, Kvistborg P, Makarov V, Havel J, et al. Cancer immunology. Mutational landscape determines sensitivity to PD-1 blockade in non-small cell lung cancer. Science. 2015;348:124-8.

19. Van Allen EM, Miao D, Schilling B, Shukla SA, Blank C, Zimmer L, et al. Genomic correlates of response to CTLA-4 blockade in metastatic melanoma. Science. 2015;350:207-11.

20. Roh W, Chen PL, Reuben A, Spencer CN, Prieto PA, Miller JP, et al. Integrated molecular analysis of tumor biopsies on sequential CTLA-4 and PD-1 blockade reveals markers of response and resistance. Sci Transl Med. 2017;9:379.

21. Chen PL, Roh W, Reuben A, Cooper ZA, Spencer CN, Prieto PA, et al. Analysis of immune signatures in longitudinal tumor samples yields insight into biomarkers of response and mechanisms of resistance to immune checkpoint blockade. Cancer Discov. 2016;6:827-37.

22. Riaz N, Havel JJ, Makarov V, Desrichard A, Urba WJ, Sims JS, et al. Tumor and microenvironment evolution during immunotherapy with Nivolumab. Cell. 2017:171:934-49 e16.

23. Newman AM, Liu CL, Green MR, Gentles AJ, Feng W, Xu Y, et al. Robust enumeration of cell subsets from tissue expression profiles. Nat Methods. 2015;12:453-7.

24. Yoshihara K, Shahmoradgoli M, Martinez E, Vegesna R, Kim H, Torres-Garcia $W$, et al. Inferring tumour purity and stromal and immune cell admixture from expression data. Nat Commun. 2013;4:2612.

25. McKenna A, Hanna M, Banks E, Sivachenko A, Cibulskis K, Kernytsky A, et al. The genome analysis toolkit: a MapReduce framework for analyzing nextgeneration DNA sequencing data. Genome Res. 2010;20:1297-303.

26. Rosenthal R, McGranahan N, Herrero J, Taylor BS, Swanton C. DeconstructSigs: delineating mutational processes in single tumors distinguishes DNA repair deficiencies and patterns of carcinoma evolution. Genome Biol. 2016;17:31.

27. Magi A, Tattini L, Cifola I, D'Aurizio R, Benelli M, Mangano E, et al. EXCAVATOR: detecting copy number variants from whole-exome sequencing data. Genome Biol. 2013;14:R120.

28. Mermel CH, Schumacher SE, Hill B, Meyerson ML, Beroukhim R, Getz G. GISTIC2.0 facilitates sensitive and confident localization of the targets of focal somatic copy-number alteration in human cancers. Genome Biol. 2011;12:R41.

29. Li Y, Xie X. Deconvolving tumor purity and ploidy by integrating copy number alterations and loss of heterozygosity. Bioinformatics. 2014;30:2121-9.

30. Anders S, Pyl PT, Huber W. HTSeq--a Python framework to work with highthroughput sequencing data. Bioinformatics. 2015;31:166-9.

31. Subramanian A, Tamayo P, Mootha VK, Mukherjee S, Ebert BL, Gillette MA, et al. Gene set enrichment analysis: a knowledge-based approach for interpreting genome-wide expression profiles. Proc Natl Acad Sci U S A. 2005:102:15545-50.

32. Roth A, Khattra J, Yap D, Wan A, Laks E, Biele J, et al. PyClone: statistical inference of clonal population structure in cancer. Nat Methods. 2014;11:396-8.

33. Chalmers ZR, Connelly CF, Fabrizio D, Gay L, Ali SM, Ennis R, et al. Analysis of 100,000 human cancer genomes reveals the landscape of tumor mutational burden. Genome Med. 2017;9:34. 
34. Bjerregaard AM, Nielsen M, Hadrup SR, Szallasi Z, Eklund AC. MuPeXI: prediction of neo-epitopes from tumor sequencing data. Cancer Immunol Immunother. 2017;66:1123-30.

35. Boegel S, Lower M, Schafer M, Bukur T, de Graaf J, Boisquerin V, et al. HLA typing from RNA-Seq sequence reads. Genome Med. 2012;4:102.

36. Sun JM. Adjuvant Durvalumab for Esophageal Cancer (Clinical Trial NCT02520453). Available at: https://www.clinicaltrials.gov/ct2/show/ NCT02520453?term=adjuvant. Accessed 5 Jun 2018.

37. Donahue JM, Nichols FC, Li Z, Schomas DA, Allen MS, Cassivi SD, et al. Complete pathologic response after neoadjuvant chemoradiotherapy for esophageal cancer is associated with enhanced survival. Ann Thorac Surg. 2009;87:392-8 discussion 8-9.

38. Okumura H, Uchikado Y, Setoyama T, Matsumoto M, Owaki T, Ishigami S, et al. Biomarkers for predicting the response of esophageal squamous cell carcinoma to neoadjuvant chemoradiation therapy. Surg Today. 2014:44:421-8.

39. Shibata T, Kokubu A, Saito S, Narisawa-Saito M, Sasaki H, Aoyagi K, et al. NRF2 mutation confers malignant potential and resistance to chemoradiation therapy in advanced esophageal squamous cancer. Neoplasia. 2011;13:864-73.

40. Motohashi H, Yamamoto M. Nrf2-Keap1 defines a physiologically important stress response mechanism. Trends Mol Med. 2004;10:549-57.

41. Schreiber RD, Old LJ, Smyth MJ. Cancer immunoediting: integrating immunity's roles in cancer suppression and promotion. Science. 2011;331: 1565-70.

42. Chen DS, Mellman I. Elements of cancer immunity and the cancer-immune set point. Nature. 2017:541:321-30.

43. Lim SH, Hong M, Ahn S, Choi YL, Kim KM, Oh D, et al. Changes in tumour expression of programmed death-ligand 1 after neoadjuvant concurrent chemoradiotherapy in patients with squamous oesophageal cancer. Eur J Cancer. 2016;52:1-9.

44. Di Maggio FM, Minafra L, Forte Gl, Cammarata FP, Lio D, Messa C, et al. Portrait of inflammatory response to ionizing radiation treatment. J Inflamm (Lond). 2015:12:14

45. Schaue D, Micewicz ED, Ratikan JA, Xie MW, Cheng G, McBride WH. Radiation and inflammation. Semin Radiat Oncol. 2015;25:4-10.

46. Barker HE, Paget JT, Khan AA, Harrington KJ. The tumour microenvironment after radiotherapy: mechanisms of resistance and recurrence. Nat Rev Cancer. 2015;15:409-25.

47. Chen DS, Mellman I. Oncology meets immunology: the cancer-immunity cycle. Immunity. 2013;39:1-10.

48. Study of Durvalumab Given With Chemoradiation Therapy in Patients With Unresectable Non-small Cell Lung Cancer (Clinical Trial NCT03519971) Available at: https://clinicaltrials.gov/ct2/show/NCT03519971. Accessed 5 Jun 2018.

49. Nivolumab or Nivolumab Plus Cisplatin, in Combination With Radiotherapy in Patients With Cisplatin-ineligible or Eligible Locally Advanced Squamous Cell Head and Neck Cancer (Clinical Trial NCT03349710). Available at: https://clinicaltrials.gov/ct2/show/NCT03349710. Accessed 5 Jun 2018.

50. Study of Pembrolizumab (MK-3475) or Placebo With Chemoradiation in Participants With Locally Advanced Head and Neck Squamous Cell Carcinoma (MK-3475-412/KEYNOTE-412) (Clinical Trial NCT03040999). Available at: https:// clinicaltrials.gov/ct2/show/NCT03040999. Accessed 5 Jun 2018.

51. Study To Compare Avelumab In Combination With Standard of Care Chemoradiotherapy (SoC CRT) Versus SoC CRT for Definitive Treatment In Patients With Locally Advanced Squamous Cell Carcinoma Of The Head And Neck (JAVELIN HEAD AND NECK 100) (ClinicalTrial NCT02952586). Available at: https://linicaltrials.gov/ct2/show/NCT02952586. Accessed 5 Jun 2018.

Ready to submit your research? Choose BMC and benefit from:

- fast, convenient online submission

- thorough peer review by experienced researchers in your field

- rapid publication on acceptance

- support for research data, including large and complex data types

- gold Open Access which fosters wider collaboration and increased citations

- maximum visibility for your research: over $100 \mathrm{M}$ website views per year

At $\mathrm{BMC}$, research is always in progress.

Learn more biomedcentral.com/submissions 